\title{
SUB-LUMINAL MOTION IN M87
}

\author{
J. A. Biretta, M. J. Reid \\ Harvard - Smithsonian Center for Astrophysics \\ W. Junor, R. Spencer, T. Muxlow \\ Nuffield Radio Astronomy Laboratories
}

The galaxy M87 (3C274, NGC4486) contains one of the nearest jet sources in the northern hemisphere, and one of our best opportunities to study a jet in detail. We have made MkII VLBI observations of the nuclear jet at $1.66 \mathrm{GHz}$ using 18 stations of the "World Array." The array included Arecibo which greatly improved the north-south resolution. The data were global fringe fitted, and self-calibrated to remove both antenna and baseline amplitude and phase errors. The resulting map (Fig. 1) has a dynamic range of 2300:1 and reveals a very complex structure. At distances $\geq \mathbf{5 0}$ mas from the core, the jet is resolved across its width and appears limb-brightened. The brightness centroid oscillates from side to side by an amount roughly equal to the jet's width; this may be caused by brightness variations at the edges of a limb-brightened jet. Within 50 mas of the core the opening angle (at the FWHM points) is $6.5^{\circ} \pm 0.3^{\circ}$; this is larger than that of the $20^{\prime \prime}$ jet, and suggests there is recollimation between the pc and kpc scales. The jet is aligned within $1^{\circ}$ of the $20^{\prime \prime}$ jet. There is no evidence for a counter-jet; the jet/counter-jet brightness ratio is $R \geq 200$.

Comparison of this map with reanalyzed data from 1980 and 1982 (Schmitt and Reid 1985) shows a prominent feature (labeled N2) moving outward at $1.1 \pm 0.3$ mas/year, or $0.34 \pm 0.09 \mathrm{c}$ for a distance of $20 \mathrm{Mpc}$. A weaker feature, N1, also shows evidence for motion. Future observations will give more accurate velocities and show whether the side to side oscillation pattern also moves outward. Sub-luminal motion has been detected in only one other active galactic nuclei, 3C84, which has similar speeds (Marr, et al. 1987). Both sources have $18 \mathrm{~cm}$ total powers which are much lower than those of the super-luminal quasars.

The observed proper motion $\beta_{\text {obs }}$ and counter-jet ratio $\mathrm{R}$ can be used to constrain the geometry and intrinsic velocity of the jet. Assuming (1) the observed feature and jet fluid move with the same speed, and (2) that there is a counter jet with an intrinsic brightness equal to that of the observed jet, we obtain limits of $\theta \leq 5.2^{\circ}$ for the angle to the line of sight, and $\beta \geq 0.8$ for the fluid speed. Such a small angle to the line of sight seems very unlikely for several reasons. (a) The enclosed solid angle is very small. (b) The deprojected opening angle for the $20^{\prime}$ jet would be $\leq 0.3^{\circ}$, which is much smaller any other radio source of similar luminosity (Bridle, 1984). And (c) the sharp feature seen in Knot A (Biretta, Owen, Hardee 1983) is difficult to obtain for small $\theta$. Together this suggests the counter-jet is either intrinsically weak or non-existent, or that the jet fluid has a much higher velocity than the observed feature (Lind and Blandford 1985). 
Bridle, A.H.: A.J. 89, 979 (1984).

\section{References}

Biretta, J.A., Owen, F.N., Hardee, P.E.: Ap.J. Lett. 274, L27 (1983).

Lind, K.R. and Blandford, R.D.: Ap.J. 295, 358 (1985).

Marr, J.M., et al.: this conference (1987).

Schmitt, J.M. and Reid, M.J.: Ap.J. 289, 120 (1985).

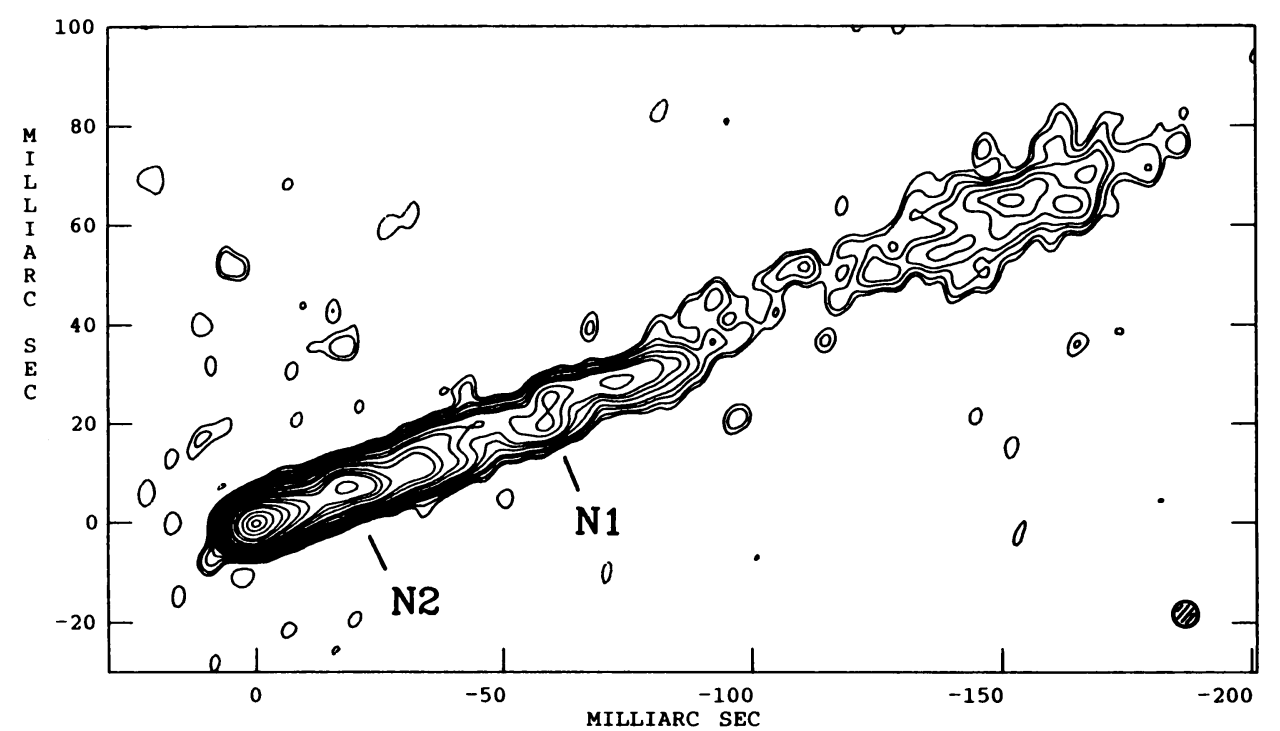

Fig. 1. Map of M87 nucleus for 1984.26. Contours are $\pm .15, .2, .3, .4, .6, .8,1,1.5,2,3$, $4,6,8,10,15,20,30,40,60,80$, and 95 percent of the peak, and the beam has a 5 mas FWHM.

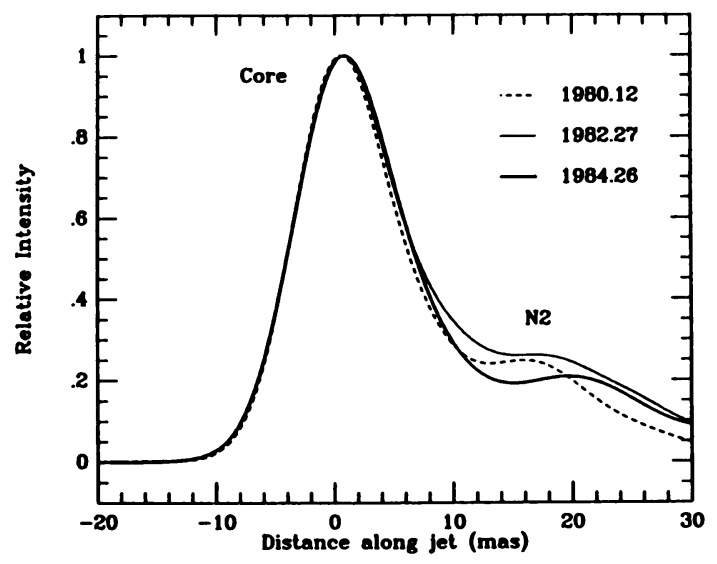

Fig. 2. Intensity profiles taken along P.A. -70 degrees. Peak intensities are normalized. All epochs have been convolved to the same 8 mas FWHM beam. 